\title{
Origin of masses in the Early Universe
}

\author{
Victor Pervushin* \\ JINR \\ E-mail: pervushetheor.jinr.ru
}

\author{
Andrej Arbuzov, Alexander Cherny, Vadim Shilin, \\ JINR, Dubna, \\ Rashid Nazmitdinov, \\ JINR, Dubna and Department de Física, Universitat de les Illes Balears, Palma de Mal- \\ lorca, Spain, \\ Alexander Pavlov, \\ Russian State Agrarian University - Moscow Timiryazev Agricultural Academy, Moscow, \\ 127550, Russia, \\ Konstantin Pichugin, \\ Kirensky Institute of Physics, Krasnoyarsk, 660036 Russia \\ Alexander Zakharov, \\ Institute of Theoretical and Experimental Physics, Moscow, 117259, Russia.
}

\begin{abstract}
New model is suggested, where the Casimir mechanism is the source of masses and conformal symmetry breaking at the Planck epoch in the beginning of the Universe. The mechanism is the Casimir energy and associated condensate, which are resulted from the vacuum postulate and normal ordering of the conformal invariant Hamiltonian with respect to the quantum elementary field operators. It is shown that the Casimir top-quark condensate specifies the value of the Higgs particle mass without involving the Higgs tachyon mass, which are put equal to zero. The Casimir mechanism yields another value of the coupling constant for the self-interaction of scalar field than the standard model does.
\end{abstract}

XXII International Baldin Seminar on High Energy Physics Problems September 15-20, 2014

JINR, Dubna, Russia

\footnotetext{
* Speaker.
} 


\section{Introduction}

The basis of modern studies on the conformal symmetry breaking at the present day epoch is the high-energy dimensional transmutation parameter [ [U, [1], founded upon the Euclidean space formulation of quantum field theory (QFT) and the renormgroup analysis. However, in the paper []], Kirzhints developed a new approach to the origin of masses in the Standard electro-weak Model in the Early Universe. Kirzhnits kept the zero harmonic of the Higgs scalar field in finite space ${ }^{1}$ and suggested the model of resurrection of the conformal symmetry at the electro-weak epoch in the beginning of the Universe.

In the present paper, we develop the Kirzhnits idea and consider the origin of masses and the conformal symmetry breaking via the scalar field zero harmonic within the Minkowskian spacetime. The Minkowskian space-time formulation, amending the Kirzhnits theory, allows us to introduce the vacuum postulate and the normal ordering of the conformal invariant action in the Early Universe.

The second difference from the Kirzhnits paper [B] is the cosmological model of the empty

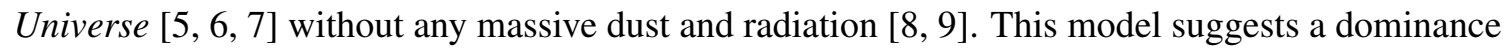
of the Casimir vacuum energy $\langle 0|\mathbf{H}| 0\rangle$, which can be calculated after corresponding regularization from the normal ordering of fields in the Hamiltonian $\mathbf{H}$ mentioned above. As is shown in the persent paper, the normal ordering leads also to the Casimir condensates of elementary fields, defined as the vacuum expectation values of the product of two elementary fields. These Casimir condensates can be sources of the masses in the Early Universe.

The third difference from the Kirzhnits paper [B] is using the conformal time-space variables (coordinates, fields, and other magnitudes) instead of the world variables. The conformal magnitudes simplify the QFT formulation. However, these magnitudes require initial data and boundary conditions that physically differ from the world initial data and boundary conditions at the beginning of the Universe. In limit of small factor $a \rightarrow 0$ we have long distance conformal intervals $d r=d \eta$, zero conformal masses $m_{\text {conf }}(a) \rightarrow 0$, and a cold empty Universe with a small conformal temperature. These conformal variables and coordinates can be treated as the real observational

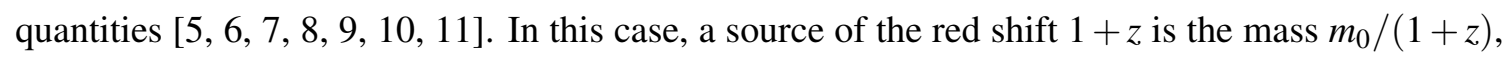
instead of the photon length $\lambda_{0} /(1+z)$ as it is accepted in the standard cosmology. In fact, observers of the modern Supernovae distances (SNe Ia data) [12] measure dimensionless number of the ratio of a photon length $\lambda_{0}$ and its units associated with the size of atom $m_{0}^{-1}$. The SNe Ia data admit both the possibilities [ $[1,6,0]$. In this paper we consider the origin of masses and condensates at the Planck epoch in the framework of the conformal cosmological model of the cold and empty Universe.

\section{The Electro-Weak Epoch Resurrection}

We shall also use the second Kirzhnits idea about the initial data of the symmetry restoration at the electro-weak epoch [B]. Using the experience of estimation of the corresponding z-factors at the instants of recombination and chemical evolution, one can suppose that the corresponding

\footnotetext{
${ }^{1}$ In agreement with the Einstein cosmological principle of 1917, this zero harmonic is the average of a scalar field over the large volume [四].
} 
$\mathrm{z}$-factor at the resurrection epoch can be at the instant. Namely, when the world CMB temperature $T_{\mathrm{w}}\left(a_{\mathrm{EW}}\right)=\frac{T_{\mathrm{CMB}}}{a_{\mathrm{EW}}}$ coincides with the present day electro-weak scale, identified with the value of t-quark mass $M_{t}=174 \mathrm{GeV}$ in the Standard cosmology variables

$$
M_{t 0}=\frac{T_{\mathrm{CMB}}}{a_{\mathrm{EW}}} \simeq 174 \mathrm{GeV}, \quad a_{\mathrm{EW}}^{-1} \equiv 1+z_{\mathrm{EW}} \simeq 0.32 \times 10^{15} .
$$

In terms of the conformal variables, the E-W scale equation (2.]) transforms to the form

$$
\left.M_{t}(a)\right|_{a=a_{\mathrm{EW}}} \equiv M_{t 0} \times a_{\mathrm{EW}}=T_{\mathrm{CMB}} .
$$

This equation describes a small particle mass in the Minkowskian space-time $d s^{2}=(d \eta)^{2}-(d x)^{2}$ at constant temperature $T_{\mathrm{CMB}}$.

In terms of the conformal variables, one can suppose that in the limit of massless particles, before the time of resurrection, the Universe was empty but not stable due to a strong interaction of the Higgs field with the cosmological scale factor. The primordial Higgs scalar particle decay becomes a source of $N_{\gamma}=\left(1+z_{\mathrm{EW}}\right)^{6} \mathrm{CMB}$ photons [ [Q] . At that time the empty Universe was filled in by the Casimir vacuum energy of all fields. The energy density of this vacuum is a single external input parameter that violates the conformal symmetry of the action. The origin of this external parameter is due to a finite volume of the Universe limited by its horizon. If at the Beginning, the Universe was quantum, one can apply the Planck's least action postulate. In this case, values of the action of the Universe are quantized. Planck's least action postulate determines the initial value of the cosmological factor $z_{\mathrm{Pl}} \sim z_{\mathrm{EW}}$ and yields the hierarchy of cosmological scales, according to their conformal weights weights $w$ and dimensions $d$ in natural units $\hbar=c=1[$, प] $]$

$$
\begin{array}{rlrl}
H_{\text {Hubble }} & \simeq H_{I} \cdot\left(1+z_{\mathrm{I}}\right)^{-2} & & d=1, w=2 \\
R_{\odot}^{-1} & \simeq H_{I} \cdot\left(1+z_{\mathrm{I}}\right)^{-1} & d=1, w=1 \\
T_{\mathrm{CMB}} & =H_{I} \cdot\left(1+z_{\mathrm{I}}\right)^{0} & & d=1, w=0 \\
M_{t 0} & \simeq H_{I} \cdot\left(1+z_{\mathrm{I}}\right)^{1} & & d=1, w=-1 \\
M_{\text {Planck }} & \simeq H_{I} \cdot\left(1+z_{\mathrm{I}}\right)^{2} & d=1, w=-2 .
\end{array}
$$

If $z_{I}=z_{\mathrm{EW}}$, one knows the present day values of the Hubble parameter $([2.3)$, the inverse size of Celestial system (2.4), the CMB temperature (2.5)), electro-weak scale (2.6), and the Planck mass (2.]), correspondingly. This hierarchy is in an agreement with a cosmological evolution of these fundamental magnitudes $F_{w, d}$. These magnitudes are defined by means of the primordial conformal Hubble parameter $H_{I}$, their conformal weights $w$, and a dimension $d$

$$
F_{w, d}(z)=\left.\gamma_{F} H_{I}^{d}\left(\frac{1+z}{1+z_{I}}\right)^{w}\right|_{z=z_{I}}=\gamma_{F} H_{I}^{d} ; \quad F_{w, d}(0)=\gamma_{F} H_{I}^{d} \cdot\left(1+z_{I}\right)^{-w}
$$

where $\gamma_{F}$ is of order of a unit. At the E-W epoch, all these values are approximately equal to the initial value of the Hubble parameter at the Planck epoch $z_{I}=z_{\mathrm{EW}} \simeq z_{\text {Planck }}$. This fact is a phenomenological support of our model of the cold empty Universe, where the modern Supernovae

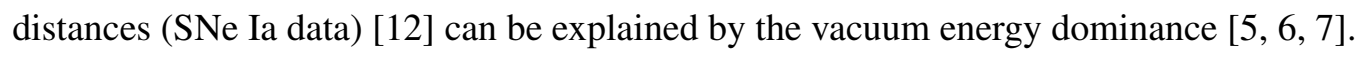




\section{Conformal Version of the Minimal Standard Model}

Now, in the framework of the condensate mechanism of conformal symmetry breaking, we consider the conformal version of SM (CSM) [[13]. In the beginning, in the CSM Lagrangian one keeps only the scalar field potential term. It gives rise to the four-type and the largest mass t-quark - Higgs field interactions

$$
L_{\mathrm{int}}=-\frac{\lambda^{2}}{8} \phi^{4}-g_{t} \phi \bar{t} t
$$

where $g_{t}=1 / \sqrt{2}$. The normal ordering of a fermion pair $f \bar{f}=: f \bar{f}:+\langle f \bar{f}\rangle$ yields the condensate density of the fermion field $\langle f \bar{f}\rangle$ in the Yukawa interaction term in Eq.(B.]). The t-quark condensate

$$
V_{\text {cond }}(\phi)=\frac{\lambda^{2}}{8} \phi^{4}-g_{t} \phi<t \bar{t}>
$$

supersedes the phenomenological negative square mass term in the Higgs potential, as a consequence of the normal ordering. At the present day $z=0$ we have a set of equations:

$$
\begin{aligned}
V_{\text {cond }}(\phi) & =\frac{\lambda^{2}}{8} \phi^{4}-g_{t} \phi\langle t \bar{t}\rangle \\
\left.\frac{d V_{\text {cond }}(\phi)}{d \phi}\right|_{\phi=v_{\text {ext }}} & =v_{\mathrm{ext}}^{3} \frac{\lambda^{2}}{2}-g_{t}\langle t \bar{t}\rangle=0 \\
\frac{d^{2} V_{\text {cond }}(\phi)}{d \phi^{2}} & =m_{h}^{2}=\frac{3 \lambda^{2}}{2} v_{\mathrm{ext}}^{2},
\end{aligned}
$$

These equations, the definition $M_{t}=g_{t} v_{\mathrm{ext}}, g_{t} \simeq 1 / \sqrt{2}$, and a present day experimental value of the Higgs particle mass $m_{h}=v_{\text {ext }} / 2$ yield a value of the t-quark condensate and the interaction constant:

$$
\frac{\langle t \bar{t}\rangle}{M_{t}^{3}}=\frac{\lambda^{2}}{2 g_{t}^{4}} \simeq \frac{1}{3}, \quad \lambda^{2}=\frac{1}{6} .
$$

This ratio of t-condensate value and the cubbed mass is close to the one at the Planck epoch, when they are determined via the primordial Hubble parameter $H_{I}$

$$
\frac{\langle t \bar{t}\rangle}{M_{t}^{3}}=\frac{\langle t \vec{t}\rangle_{I}}{H_{I}^{3}} \simeq \frac{1}{3}
$$

as it was calculated in the book [प]]) (see pp. 323-325) in according to the equation ([2.8)).

\section{Conclusion}

We suggested to induce a spontaneous conformal symmetry breaking in the Standard Model by means of the quantum anomalies. This enables us to avoid the problem of the regularization of the divergent tadpole loop integrals in the Coleman-Weinberg mechanism of a dimensional transmutation, by relating them to condensate values. These values could be hopefully extracted from experimental observations. The top quark condensate can supersede the tachyon-like mass term in the Higgs potential. The suggested mechanism allows to establish relations between condensates and masses, including the Higgs boson one. In a sense, we suggest a simple bootstrap between 
the Higgs and top fields (and their condensates). We consider the Higgs boson to be an elementary particle, without introduction of any additional interaction beyond the SM, contrary to various technicolor models. After the spontaneous symmetry breaking in the tree level Lagrangian, the difference from the SM appears only in the value of the Higgs boson self-coupling $\lambda$. The latter hardly can be extracted from the LHC data, but it will be certainly measured at a future linear $e^{+} e^{-}$ collider.

\section{References}

[1] S. Coleman, R. Jackiw. Ann. Phys. 67 (1971) 552.

[2] S. Coleman, R. Weinberg. Phys. Rev. 7 (1973) 1888.

[3] D.A. Kirzhnits, ZHETF Letters. 15 (1972) 745.

[4] A. Einstein. Sitzungsber. Preuss. Akad. Wiss. Berlin (Math. Phys.) (1917) 142.

[5] D. Behnke, D. Blaschke, V. Pervushin, and D. Proskurin. Phys. Lett. B 530 (2002) 20.

[6] A.F. Zakharov, V.N. Pervushin. Int. J. Mod. Phys. D 19 (2010) 1875. [arXiv: 1006.4745 [gr-qc]]

[7] A.F. Zakharov, V.N. Pervushin. Nuclear Physics. 75 (2012) 1492.

[8] A.B. Arbuzov, B.M. Barbashov, R.G. Nazmitdinov, V.N. Pervushin, A. Borowiec, K.N. Pichugin, A.F. Zakharov. Phys. Lett. B 691 (2010) 230.

[9] V.N. Pervushin, A.B. Arbuzov, B.M. Barbashov, R.G. Nazmitdinov, A. Borowiec, K.N. Pichugin, A.F. Zakharov. Gen. Relativ. Gravit. 44 (2012) 2745.

[10] B.M. Barbashov, V.N. Pervushin, A.F. Zakharov, V.A. Zinchuk. Phys. Lett. B 633 (2006) 458. [arXiv: hep-th/0501242]

[11] Victor Pervushin and Alexander Pavlov, (2014) "Principles of Quantum Universe", 480 pp. LAP LAMBERT Academic Publishing, Saarbrücken, Germany, ISBN 978-3-659-50742-7.

[12] A.G. Riess, et al. [Supernova Search Team Collaboration]: Astrophys. J. 560 (2001) 49. [arXiv: astro-ph/0104455]

[13] V.N. Pervushin, A.B. Arbuzov, R.G. Nazmitdinov, A.E. Pavlov, A.F. Zakharov. arXiv:1209.4460 [hep-ph].

[14] V. Pervushin, A. Arbuzov, B. Barbashov, A. Cherny, A. Dorokhov, A. Borowiec, R. Nazmitdinov, A. Pavlov, V. Shilin, A. Zakharov. PoS (Baldin ISHEPP XXI) 023. International Baldin Seminar on High Energy Physics Problems (2012). http://pos.sissa.it

[arXiv: hep-ph/1211.4386] 